\title{
The floristic compositions of vascular epiphytes of a seasonally inundated forest on the coastal plain of Ilha do Mel Island, Brazil
}

\author{
Rodrigo de Andrade Kersten \& Sandro Menezes Silva \\ Pontifícia Universidade Católica do Paraná (PUC-PR), Centro de Ciências Biológicas e da Saúde (CCBS). Rua \\ Imaculada Conceição, 1155 - Prado Velho - Curitiba - PR - BR. CEP: 80215-901. kersten@pop.com.br
}

Received 13-I-2003. Corrected 16-IV-2004. Accepted 08-VII-2005.

\begin{abstract}
A $3000 \mathrm{~m}^{2}$ area of seasonally inundated forest on the island of Ilha do Mel (2530" S 48 $23^{\circ}$ ” W) in Paraná, Brazil, was sampled by collecting plants from all strata, using climbing equipment when necessary. The area harbors 103 species of epiphytes, in 49 genera and 20 families, of which 28 species are pteridophytes and 75 magnoliophytes (64 Liliopsida, 11 Magnoliopsida). The most common families are Orchidaceae, Bromeliaceae, Polypodiaceae and Araceae, and frequent genera are Vriesea, Epidendrum, Maxillaria, Pleurothallis and Prosthechea. Eight families were represented by one species each. Most species were classified as obligatory holoepiphytes (62\%), followed by the relatively more rare preferential holoepiphytes (13\%), facultative epiphytes $(11 \%)$, hemiepiphytes $(9 \%)$ and accidental epiphytes (6 \%). Rev. Biol. Trop. 54 (3): 935-942. Epub 2006 Sept. 29.
\end{abstract}

Key words: Brazil, Atlantic rain forest, coastal plain forest, floristic composition, Orchidaceae, Restinga, vascular epiphytes.

Epiphytes comprise a significant proportion of the plant diversity of tropical forests and may represent as much as $50 \%$ of the plant species in these forests (Gentry and Dodson 1987b). Humid tropical forests are exceptionally notable due to the richness and abundance of epiphytes (Madison 1977). Diversity and richness of epiphytic orchids, for example, is great in the tropics and declines rapidly as the latitude approaches $30^{\circ}$, which is the limit of the influence of tropical air masses (Waechter 1998b). However, many endemic temperate epiphytes occur in the Southern Hemisphere, in strong contrast to the Northern Hemisphere, where mostly outlying species representing large tropical genera occurs (Benzing 1990).

While several studies have examined epiphyte floristic in Brazil (Gottsberger and Morawetz 1993 in the Amazon; Aguiar et al. 1981, Cervi and Dombrowski 1985, Cervi et al. 1988, Waechter 1992, 1998a, Dittrich et al. 1999, Kersten and Silva 2002 in subtropical forests; Pinto et al. 1995, Dislich and
Mantovani 1998 in seasonal forests; Waechter 1986, 1992, Fontoura et al. 1997, Labiak and Prado 1998, Piliackas et al. 2000, Kersten and Silva 2001 in tropical formations), this study addresses the epiphytic community of a seasonally inundated coastal plain forest on the island of Ilha do Mel in the state of Paraná, in southern Brazil. This forest, has close affinities with the threatened and poorly studied Atlantic Coastal Forest of southeastern Brazil, and is an area of endemism for many plant and animal species (Pimm 2001). Identification of epiphytic species in this forest provides the first step in understanding their distribution and abundance as well as paving the way for further studies of the ecology and evolution of this community.

\section{MATERIALS AND METHODS}

The study area comprises $3000 \mathrm{~m}^{2}$ of the coastal plain of the island Ilha do Mel, in the state of Paraná, Brazil. Flooded during the 
rainy seasons, this relatively closed forest contains three main strata: the lower stratum contains herbaceous plants, leaf litter and shoots, the middle stratum bushes and samplings, and the upper stratum, which may reach over $20 \mathrm{~m}$, with few dominant species (Silva 1998).

Plants were collected from all strata, using climbing equipment when necessary (Ingram and Lowman 1995), samples of each fertile species were collected as a reference collection to be maintained in herbaria. Collected epiphytes were identified by specialist, using published literature, or by comparison with herbaria specimens in the UPCB (Herbarium of the Botany Department at the Federal University of Paraná) and MBM (Herbarium of the Municipal Botanical Museum). All the material gathered was prepared for and placed in the UPCB. Floristic similarity was compared among sites using the Jaccard Similarity Index.

Taxonomy followed Cronquist (1988) for Magnoliophyta and Moran (1995) for Pteridophyta. Epiphytes, based on their observed relationship with their host plant, were characterized as obligatory holoepiphyte, preferential holoepiphytes, facultative holoepiphyte, accidental holoepiphyte, primary and secondary hemiepiphyte, based on Benzing (1990).

\section{RESULTS}

The many species $(n=103)$ of epiphytes were distributed among 20 families, in 49 genera. Pteridophyta contributed 10 families, 15 genera and 28 species. The rest were within the Magnoliophyta, and included 10 families: Liliopsida contained three families, with 27 genera and 64 species; Magnoliopsida contained seven families, with seven genera and 11 species. The most speciose families were Orchidaceae (18 genera and 42 species), Bromeliaceae (six genera and 16 species), Polypodiaceae (five genera with 11 species), and Araceae (three genera and six species). Eight families were represented by one species each (four dicots and four pteridophytes, Table 1).
Seven species classified as accidental epiphytes or climbing lianas, (Marcgravia polyantha, Norantea brasiliensis - Marcgraviaceae, Polybotrya cylindrica - Dryopteridaceae, Prunus mirtyfolia - Rosaceae, Mikania sp. - Asteraceae and Conomorpha peruviana Myrsinaceae) were not included in the species list discussed here in, even though similar species were included in epiphyte checklist by Ingram et al. (1996) and Nadkarni (1986).

The most species rich genera were Vriesea (Bromeliaceae) and Maxillaria (Orchidaceae), each with eight species. Next was Epidendrum (Orchidaceae) with six species, Pleurothallis (Orchidaceae) with five species and Trichomanes (Hymenophyllaceae) and Prosthechea (Orchidaceae) with four species each. Five genera had three species each (Elaphoglossum, Octomeria, Campyloneurum, Polypodium and Tillandsia), and 11 genera had two species, while the remaining 28 genera were each represented by one species. The epiphytic categories were distributed among obligatory holoepiphytes (62\%, including all of the Orchidaceae), preferential holoepiphytes (13\%), facultative epiphytes (11\%), hemiepiphytes (9\%) and accidental holoepiphytes (6\%).

\section{DISCUSSION}

A total of 201 vascular plant species have been identified to date in the study area (M.C.M. Marques pers. com., Silva 1998), of which $51 \%$ (103 spp.) are epiphytes and $41 \%$ (86 spp.) are characteristic holoepiphyte. Indeed, over $59 \%$ of the species are canopy dependent (including climbing lianas). This high proportion of epiphytes is greater than that reported in any other forest studied (Gentry and Dodson 1987a). In Jamaica (Kelly 1985) only $25 \%$ of the total flora were epiphytes, in La Selva they comprise $25 \%$ and in Centinela Ridge this proportion grows to $35 \%$ (Both in Gentry and Dodson 1987b), in Costa Rica (Ingram et al 1996) $25 \%$ of total species were expected to grow epiphytically and in Macaé de Cima (Fontoura et al. 1997) $27 \%$ of the 
TABLE 1

Species list for this study, followed by epiphyte type and catalog number in the UPCB herbarium

\begin{tabular}{|c|c|c|}
\hline $\begin{array}{l}\text { FAMILY } \\
\text { Species }\end{array}$ & Type & UPCB \\
\hline \multicolumn{3}{|l|}{ ARACEAE } \\
\hline Anthurium itanhaense Engl. & HMSF & 43154 \\
\hline $\begin{array}{l}\text { Anthurium pentaphyllum (Aubl.) G. } \\
\text { Don }\end{array}$ & HMS & 38556 \\
\hline Anthurium scandens (Aubl.) Engl. & HLC & 43097 \\
\hline Monstera adansonii Schott & HMS & 43100 \\
\hline $\begin{array}{l}\text { Philodendron bipinnatifidum Schott } \\
\text { ex Endl. }\end{array}$ & HMP & 41893 \\
\hline Philodendron corcovadense Kunth & HMS & 43155 \\
\hline \multicolumn{3}{|l|}{ ASPLENIACEAE } \\
\hline Asplenium serra Langsd. \& Fisch. & HLA & 25795 \\
\hline \multicolumn{3}{|l|}{ BLECHNACEAE } \\
\hline Blechnum serrulatum Rich. & HLA & 23761 \\
\hline \multicolumn{3}{|l|}{ BROMELIACEAE } \\
\hline Aechmea nudicaulis (L.) Griseb. & HLF & 43112 \\
\hline Aechmea ornata Baker. & HLF & 40563 \\
\hline Bromelia antiacantha Bertol. & HLA & 13940 \\
\hline $\begin{array}{l}\text { Catopsis sessiliflora (Ruiz \& Pav.) } \\
\text { Mez. }\end{array}$ & HLF & 41712 \\
\hline Nidularium innocentii Lem. & HLF & 43113 \\
\hline Tillandsia gardneri Lindl. & HLC & 27323 \\
\hline Tillandsia geminiflora Brongn. & HLC & 43111 \\
\hline Tillandsia tenuifolia $\mathrm{L}$. & HLC & 31877 \\
\hline Vriesea atra $\mathrm{Mez}$ & HLC & 30457 \\
\hline Vriesea carinata Wawra & HLC & 43115 \\
\hline Vriesea gigantea Mart. ex Schult. f. & HLC & 22859 \\
\hline Vriesea philippocoburgii Wawra & HLF & 30444 \\
\hline $\begin{array}{l}\text { Vriesea procera (Mart. ex Schult.f.) } \\
\text { Wittm. }\end{array}$ & HLC & 43115 \\
\hline Vriesea rodigasiana E.Morren & HLF & 43135 \\
\hline Vriesea scalaris E. Morren & HLC & 43110 \\
\hline Vriesea vagans (L.B. Sm.) L.B. Sm. & HLF & 30445 \\
\hline \multicolumn{3}{|l|}{ CACTACEAE } \\
\hline Rhipsalis cereuscula Haw. & HLC & 42361 \\
\hline Rhipsalis teres (Vell.) Steud & HLC & 40823 \\
\hline \multicolumn{3}{|l|}{ CECROPIACEAE } \\
\hline Coussapoa microcarpa (Schott) Rizzini & HMP & 15064 \\
\hline
\end{tabular}

FAMILY

Species

CLUSIACEAE

Clusia criuva Cambess.

HMPF 24873

DAVALLIACEAE

Nephrolepis biserrata (Sw.) Schott

HLA 30440

Nephrolepis rivularis (Vahl.) Mett.

ex Krug

HLA 31275

DENNSTAEDTIACEAE

Lindsaea quadrangularis Raddi

HLA $\quad 43134$

\section{DRYOPTERIDACEAE}

Rumohra adiantiformis (G. Forst) Ching

HLF 13446

\section{GESNERIACEAE}

Codonanthe devosiana Lem.

HLC

15037

Codonanthe gracilis (Mart.) Hanst.

HLC 43102

HYMENOPHYLACEAE

Trichomanes cristatum Kaulf.

HLC 43133

Trichomanes hymenoides Hedw.

HLC 43108

Trichomanes krauzii Hook \& Greeg.

HLC 43136

Trichomanes pyxidiferum $\mathrm{L}$.

HLC 43107

LOMARIOPSIDACEAE

Elaphoglossum crassinerve Moor

HLC 31301

Elaphoglossum lingua (C. Presl) Brack.

HLC 43106

Elaphoglossum subarborescens Rosent.

HLC 43105

\section{LYCOPODIACEAE}

Huperzia flexibilis (Fée) B.Øllg.

HLC 25834

Huperzia mandiocana (Raddi) Trevis.

HLC 43132

MORACEAE

Ficus luschnathiana (Miq.) Miq.

HMPF 40994

\section{ORCHIDACEAE}

Campylocentrum linearifolium Schltr. ex Mansf.

HLC $\quad 43151$

Cattleya forbesii Lindl.

HLC 43117

Dichaea anchorifera Cogn.

HLC 43096

Epidendrum latilabre Lindl.

HLC 43146

Epidendrum paniculatum Ruiz et Pavan

HLC $\quad 13919$

Epidendrum ramosum Jacq.
HLC 40495 
TABLE 1 (Continued)

Species list for this study, followed by epiphyte type and catalog number in the UPCB herbarium

\begin{tabular}{|c|c|c|c|c|c|}
\hline $\begin{array}{l}\text { FAMILY } \\
\text { Species }\end{array}$ & Type & UPCB & $\begin{array}{l}\text { FAMILY } \\
\text { Species }\end{array}$ & Type & UPCB \\
\hline Epidendrum rigidum Jacq. & HLC & 43142 & \multirow{2}{*}{$\begin{array}{l}\text { Prosthechea vespa (Vell.) } \\
\text { W.E.Higgins }\end{array}$} & \multirow{2}{*}{ HLC } & \multirow{2}{*}{43139} \\
\hline Epidendrum strobiliferum Rchb. f. & HLC & 22839 & & & \\
\hline Jacquiniella globosa (Jacq.) Schltr. & HLC & 8279 & $\begin{array}{l}\text { Reichenbachanthus reflexus (Lindl.) } \\
\text { C.Porto \& Brade }\end{array}$ & HLC & 43145 \\
\hline Lankesterella ceracifolia Ames & HLC & 14551 & \multirow{2}{*}{$\begin{array}{l}\text { Scaphyglottis modesta (Rchb. f.) Schltr. } \\
\text { Stelis fraterna Lindl. }\end{array}$} & HLC & 43141 \\
\hline Lockhartia lunifera Rchb.f. & HLC & 36407 & & HLC & 43148 \\
\hline Maxillaria brasiliensis Brieb.\& Illg & HLC & 43144 & Stelis sp. & HLC & 37380 \\
\hline Maxillaria chlorantha Lindl. & HLC & 43149 & Trigonidium latifolium Lindl. & \multirow[t]{2}{*}{ HLC } & \multirow[t]{2}{*}{43119} \\
\hline Maxillaria imbricata Barb.Rodr. & HLC & 43610 & PIPERACEAE & & \\
\hline Maxillaria marginata Fenzl. & HLC & 40529 & Peperomia emarginella (Sw. ex & \multirow{2}{*}{ HLC } & \multirow{2}{*}{40824} \\
\hline Maxillaria parahybunensis Cogn. & HLC & 43608 & Wikstr.) C. DC. & & \\
\hline Maxillaria rodriguesii Cogn. & HLC & 42787 & Peperomia glabella (Sw.) A.Dietr. & HLF & 43120 \\
\hline Maxillaria rufescens Lindl. & HLC & 43156 & Peperomia urocarpa Fisch. \& C.A. Mey. & HLF & 43101 \\
\hline Maxillaria sp. 1 & HLC & -- & \multicolumn{3}{|l|}{ POLYPODIACEAE } \\
\hline Octomeria crassifolia Lindl. & HLC & 40107 & Campyloneurum acrocarpon Fée & HLC & 43130 \\
\hline Octomeria fibrifera Schltr. & HLC & 32124 & Campyloneurum nitidum C. Presl. & HLC & 43128 \\
\hline Octomeria gracilis Barb. Rodr. & HLC & 43118 & Campyloneurum rigidum J.Sm. & HLC & 43129 \\
\hline Oncidium ciliatum Lindl. & HLC & 47305 & \multirow{2}{*}{$\begin{array}{l}\text { Microgramma percussa (Cav.) de } \\
\text { la Sota }\end{array}$} & \multirow{2}{*}{ HLC } & \multirow{2}{*}{43121} \\
\hline Oncidium uniflorum Booth. & HLC & 43140 & & & \\
\hline Physosiphon spiralis Lindl. & HLC & 43147 & $\begin{array}{l}\text { Microgramma vacciniifolia (Langsd. } \\
\text { \& Fisch.) Copel }\end{array}$ & HLC & 43124 \\
\hline Pleurothallis corticicola Schltr. & HLC & 47649 & \multirow{3}{*}{$\begin{array}{l}\text { Pecluma recurvata (Kaulf.) M.G.Price } \\
\text { Pleopeltis pleopeltifolia (Raddi) } \\
\text { Alston }\end{array}$} & \multirow[t]{2}{*}{ HLC } & \multirow[t]{2}{*}{39044} \\
\hline Pleurothallis marginalis $\mathrm{Rchb} . \mathrm{f}$. & HLC & 39863 & & & \\
\hline Pleurothallis matinhensis Hoehne & HLC & 43098 & & HLC & 43122 \\
\hline Pleurothallis saundersiana Rchb.f. & HLC & 43138 & Pleopeltis astrolepis (Liebm.) E. Fourn. & HLC & 43127 \\
\hline Pleurothallis seriata Lindl. & HLC & 43153 & \multirow{2}{*}{$\begin{array}{l}\text { Polypodium catharinae Langsd.\& } \\
\text { Fisch. }\end{array}$} & \multirow{2}{*}{ HLC } & \multirow{2}{*}{43013} \\
\hline Polystachya caespitosa Barb.Rodr. & HLC & 43150 & & & \\
\hline Polystachya concreta (Jacq.) Garay \& & HLC & 43143 & Polypodium chnoophorum Kunze & HLC & 31311 \\
\hline HR Sweet & $\mathrm{HLC}$ & 43143 & Polypodium hirsutissimum Raddi & HLC & 43125 \\
\hline Prosthechea fragrans (Sw.) W.E.Higgins & HLC & 43116 & RUBIACEAE & & \\
\hline $\begin{array}{l}\text { Prosthechea inversa (Lindl.) } \\
\text { W.E.Higgins }\end{array}$ & HLC & 37392 & Hillia parasitica Jacq. & HMS & 40729 \\
\hline Encyclia patens Hook & HLC & 43099 & & & \\
\hline Prosthechea pygmaea (Hook) & HLC & 43152 & $\begin{array}{l}\text { Radiovittarla stlpitata (Kuntze) E.H. } \\
\text { Crane }\end{array}$ & HLC & 43131 \\
\hline & & & Vittaria lineata (L.) Sm. & HLC & \\
\hline
\end{tabular}

$(\mathrm{HL}=$ holoepiphyte, $\mathrm{HM}=$ hemiepiphyte, $\mathrm{O}=$ obligatory, $\mathrm{P}=$ preferential, $\mathrm{F}=$ facultative, $\mathrm{A}=$ accidental, $\mathrm{P}=$ primary, $\mathrm{S}=$ secondary) 
flora was epiphytic. This epiphyte quotient (Hosokawa 1950) is even greater than the presented in Engwald (2000) who found that the 191 species documented comprises $45 \%$ of total flora species. Nearby in Brazil, in an Araucaria forest, epiphytes constitute $22 \%$ of the flora (Dittrich et al. 1999). Nearby, in a seasonal forest in Argentina, epiphytes were reported to be represented by fewer species than were trees, while no precise numbers were provided (Brown 1990). Hence, the flora of Ilha do Mel includes surprisingly many epiphytes, and supports the premise that characteristic epiphytes are an important part of the tropical forest plant communities (Madison 1977), and that they are responsible for an important part of the diversity of these complex ecosystems (Gentry and Dodson 1987b).

The four most species rich families (Orchidaceae, Bromeliaceae, Polypodiaceae, Araceae) together are represented here by 75 species being the largest families elsewhere (Benzing 1990, Gentry and Dodson 1987b, Kress 1986, Madison 1977). Orchidaceae that worldwide represents $10 \%$ of the total terrestrial vascular flora (Atwood 1986) represents $40 \%$ of the epiphytic and $20 \%$ of the total species on our work. The absence of the family Ericaceae in this study as in many others areas in Brazil (Cervi and Dombrowski 1985, Cervi et al. 1988, Waechter 1986, 1992, 1998a, Fontoura et al. 1997, Dislich and Mantovani 1998, Dittrich et al. 1999, Kersten and Silva 2001, 2002) should be surprising according to Kress (1986), given that it is a common family in Central America (Sudgen and Robins 1979, Nadkarni 1986, Ingram et al. 1996).

Only two areas studied in Brazil had greater species-richness than that presented here (Table 2). One study (Torres, Waechter 1986) is located approximately $400 \mathrm{~km}$ south of Ilha do $\mathrm{Mel}$ at $30^{\circ} \mathrm{S}$. This region is described as a transition zone (Takhtajan 1986), at the interface of seasonal forests and mixed rainforests, and so contains species that are typical of both (eg Blechnum binervatum - Blechnaceae, Tillandsia stricta and T. mallemontii - Bromeliaceae) and of Atlantic Forests (eg Hymenophyllum vestitum in the family Hymenophyllaceae).
The second (Fontoura et al. 1997) located on Rio de Janeiro is much larger and includes montana and altomontana forest having a high ecosystem diversity and so a higher species diversity. The area studied here is more species-rich than any other coastal plains in southern Brazil (Kersten and Silva 2001), any other area outside of the influence of the Atlantic Ocean in southern Brazil (Cervi and Dombrowski 1985, Cervi et al. 1988, Dislich and Mantovani 1998, Dittrich et al. 1999, Kersten and Silva 2002), as well as areas under the influence of temperate air masses (Aguiar et al. 1981, Waechter 1998a). This pattern is probably due to the tropical origins of the epiphytic component of this forest. Four study sites in Central America (Kelly 1995, Nadkarni et al. 1995, Ingram et al. 1996, Engwald et al. 2000) had greater species-richness than this study but all of them had a much greater study area, varying from 5 times greater (1.5 ha in Engwald et al. 2000) to more than 13 times greater (4 ha in Nadkarni et al. 1995, Ingram et al. 1996).

The epiphytic community of Ilha do Mel (here and Kersten and Silva 2001) was most similar (Table 2) to Torres (Waechter 1986, 1992) and Ubatuba (Piliackas et al. 2000), and less so with Macaé de Cima (Fontoura et al. 1997) and Montenegro and Triunfo (Aguiar et al. 1981). Torres and Ubatuba owe their similarities to their similar formations and their proximity with Ilha do Mel. Macaé was less similar to Ilha do Mel due to its greater number of species, and Montenegro and Triunfo due to fewer species.

Holoepiphytes formed the vast majority of species here, a trend that is apparently common (Kelly 1985, Pinto et al. 1995, Dittrich et al. 1999, Kersten and Silva 2001, 2002). Facultative epiphytes, with $10-15 \%$ of the total, form the second most important group of epiphytes. In this second group, Aechmea nudicaulis, Codonanthe gracilis, Peperomia glabella, and Clusia criuva were often found as free living plants as well as epiphytes (Silva 1998). Hemiepiphytes comprised nine species, with three being primary hemiepiphytes (Clusia criuva, Ficus cf. luschnathiana and Coussapoa microcarpa) and the remainder were secondary epiphytes. 
TABLE 2

Epiphyte specie richness in Brazilian studies

Location/Reference

Ilha do Mel, Paranaguá, PR, 2530'S, $48^{\circ} 23^{\prime} \mathrm{W}$ this study

Ilha do Mel, Paranaguá, PR, 2530'S, $48^{\circ} 23^{\prime}$ W Kersten and Silva 2001

Macaé de Cima, RJ, 22²3'S, 4247'W Fontoura et al. 1997

Torres I and II, RS, $29^{\circ} 21^{\prime} \mathrm{S}, 49^{\circ} 45^{\prime} \mathrm{W}$ Waechter 1986, 1992

Curitiba, PR, 2525'S, 49 $18^{\prime}$ 'W Dittrich et al. 1999

UFPR, 2525'S, 49¹8'W Cervi et al. 19851988

Osório, RS, 2958'S, 50¹4'W Waechter 1998

USP, 233' $\mathrm{S}, 46^{\circ} 43^{\prime} \mathrm{W}$ Dislich and Mantovani 1998

Estação Ecológica do Taim, RS, 32³3'S, 55²6’W Waechter 1992

Montenegro/Triunfo, RS, 2950’S, 51²7’W Aguiar et al. 1981

$\begin{array}{ccc}\text { \# of Species } & \text { Forest Type } & \text { Area } \\ 103 & \text { DR } & 0.3 \\ 77 & \text { DR } & 0.3 \\ 306 & \text { DR } & \text { n.r. } \\ 115 \text { (I) } 93 \text { (II) } & \text { DR } & \text { n.r. } \\ 72 & \text { MR } & 50 \\ 32 & \text { MR } & \text { n.r. } \\ 53 & \text { SF } & 1 \\ 38 & \text { SF } & 0.2 \\ 24 & \text { SF } & \text { n.r. } \\ 17 & \text { SF } & \text { n.r. }\end{array}$

Forest type is abbreviated as follows: $\mathrm{MR}=$ Mixed Rainforest, $\mathrm{DR}=$ Dense Rainforest, $\mathrm{SF}=\mathrm{Seasonal}$ Forest. Area is in hectares, n.r. indicates the study did not report area.

Seven other species were accidental epiphytes, mentioned here but not considered in comparisons of communities. Examples are Bromelia antiacantha in spite of its usually terrestrial life style, was found as an epiphyte (see Aguiar et al. 1981, Waechter 1998a). Two species normally found as terrestrial trees (Prunus myrtifolia and Conomorpha peruviana) and one vine (Micania sp.) were also observed as epiphytes. It is unknown whether these accidental epiphytes may survive to reproduce as epiphytes. Perhaps they represent the type of transition from free-living plant to the epiphytic habit that may have been important in the evolution of some epiphytic plants.

In summary, the vascular epiphyte component of this plant community in southern Brazil comprises over half $(51 \%)$ of the vascular plant species in the community. Orchidaceae and Bromeliaceae are the two most species-rich families. While most species are characteristic holoepiphytes, $25 \%$ of this community is divided between hemiepiphytes, facultative holoepiphytes and accidental epiphytes.

\section{RESUMEN}

Se muestreó plantas de todos los estratos en una área de $3000 \mathrm{~m}^{2}$ de selva estacionalmente inundada en Ilha do Mel (2530 “S 4823' W), Paraná, Brazil. El área tiene 103 especies de epífitas en 49 géneros y 20 familias, de las cuales 28 especies son pteridófitas y 75 magnoliófitas (64 Liliopsida, 11 Magnoliopsida). Las familias más comunes son Orchidaceae, Bromeliaceae, Polypodiaceae y Araceae, y los géneros más frecuentes Vriesea, Epidendrum, Maxillaria, Encyclia y Pleurothallis. Ocho familias solamente están representadas por una especie cada una. La mayoría de las especies son loepífitas obligadas (62\%), seguidas de las relativamente escasas holoepífitas preferenciales $(13 \%)$, epífitas facultativas $(11 \%)$, hemiepífitas (9\%) y epífitas accidentales (6\%).

Palabras clave: Brazil, selva lluviosa atlántica, selva de planicie costera, composición florística, Orchidaceae, Restinga, epifitas vasculares.

\section{REFERENCES}

Aguiar, L.W., V. Citadini-Zanette, L. Martau \& A. Backes. 1981. Composição florística de epífitos vasculares numa area localizada nos municípios de Montenegro 
e Triunfo, Rio Grande do Sul, Brazil. Iheringia 28: $55-93$.

Atwood, J.T. 1986. The size of Orchidaceae and the systematic distribution of epiphytic orchids. Selbyana 9: 171-186.

Benzing, D.H. 1990. Vascular epiphytes. Cambridge University, Cambridge, England.

Brown, D.A. 1990. El epifitismo en las selvas montanas del Parque Nacional "El Rey" Argentina: Composición florística y padrón de distribución. Rev. Biol. Trop. 38: $155-166$.

Cervi, A.C. \& L.T.D. Dombrowski. 1985. Bromeliaceae de um capão de floresta primária do Centro Politécnico de Curitiba (Paraná, Brazil). Fontqueria 9: 09-11.

Cervi, A.C., L.A. Acra, L. Rodrigues, S. Train, S.L Ivachechen \& A.L.O.R. Moreira. 1988. Contribuição ao conhecimento das epífitas (exclusive Bromeliaceae) de uma floresta de araucária do primeiro planalto paranaense. Ínsula 18: 75-82.

Cronquist, A. 1988. The evolution and classification of flowering plants. The New York Botanical Garden, New York, USA.

Dislich, R. \& W. Mantovani. 1998. Flora de epífítas vasculares da Reserva da Cidade Universitária Armando de Salles Oliveira (São Paulo, Brazil). Bol. Bot. Univ. S. Paulo 17: 61-83.

Dittrich, V.A.O., C. Kozera \& S.M. Silva. 1999 Levantamento florístico de epífitos vasculares no Parque Barigüi, Paraná, Brazil. Iheringia 52: 11-22.

Engwald, S., V. Schimit-Neuerburg \& W. Barthlott. 2000. Epiphytes in rain forest of Venezuela - diversity and dynamics of a biocenosis, p. 425-434. In S.W. Breckle, B. Schweizer \& U. Arndt (eds.). Results of worldwide ecological studies. Proceedings of the first symposium by the AFW Foundation. Günter Heimbach, Hoheneim, Holland.

Fontoura, T., L.S. Sylvestre, A.M.S. Vaz \& C.M. Vieira 1997. Epífitas vasculares, hemiepífitas e hemiparasitas da Reserva Ecológica de Macaé de Cima, p. 89101. In H.C. Lima \& R.R. Guedes-Bruni (eds.). Serra de Macaé de Cima: diversidade florística e conservação da Mata Atlântica. Editora do Jardim Botânico do Rio de Janeiro, Rio de Janeiro, Brasil.

Gentry, A.H. \& C.H. Dodson. 1987a. Contribution of non-trees to species richness of a tropical rain forest. Biotropica 19: 149-156.

Gentry, A.H. \& C.H. Dodson. 1987b. Diversity and biogeography of neotropical vascular epiphytes. Ann. Miss. Bot. Gar. 74: 205-233.
Gottsberger, G. \& W. Morawetz. 1993. Development and distribution of the epiphytic flora in an Amazonian savanna in Brazil. Flora 188: 145-151.

Hosokawa, T. 1950. Epyphyte-quotient. Bot. Mag. Tokyo 63: 18-19.

Ingram, S.W. \& M.D. Lowman. 1995. The collection and preservation of plant material from the tropical forest canopy. p. 5-15. In M.D. Lowman \& N.M. Nadkarni (eds.). Forest Canopies Academic, San Diego, California, USA.

Ingram, S.W., A. Ferrell, K. Ingram \& N.M. Nadkarni. 1996. Floristic composition of vascular epiphytes in a neotropical cloud forest, Monteverde, Costa Rica. Selbyana 17: 88-103.

Kelly, D.L. 1985. Epiphytes and climbers of a Jamaican rain forest: vertical distributions, life forms and life history. J. Biogeogr. 2: 233-243.

Kersten, R.A. \& S.M. Silva. 2001. Composição florística e distribuição espacial de epífitas vasculares em floresta da planície litorânea da Ilha do Mel, Paraná, Brazil. Rev. Bras. Bot. 24:213-226.

Kersten, R.A. \& S.M. Silva. 2002. Florística e estrutura do componente epifítico vascular em floresta ombrófila mista aluvial do rio Barigüi, Paraná, Brazil. Rev. Bras. Bot. 25: 259-267

Kress, W.J. 1986. The systematic distribution of vascular epiphytes: an update. Selbyana 9: 2-22.

Labiak, P.H. \& J. Prado. 1998. Pteridófitas epífitas da Reserva Volta Velha, Itapoá - Santa Catarina, Brazil. Bol. Inst. Bot. 11: 1-79.

Madison, M. 1977. Vascular epiphytes: their systematic occurrence and salient features. Selbyana 2: 1-13.

Moran, R.C. 1995. Clave para las familias de pteridofitas. Flora Mesoamericana. Vol. 1: Psilotaceae a Salviniaceae. UNAM, Mexico, DF, Mexico.

Nadkarni, N.M. 1986. An ecological overview and checklist of vascular epiphytes in the Monteverde cloud forest reserve, Costa Rica. Brenesia 24: 55-632.

Nadkarni, N.M., T.J. Matelson \& W.A. Haber. 1995. Structural characteristics \& floristic composition of a Neotropical cloud forest, Monteverde, Costa Rica. J. Trop. Ecol. 11:481-495.

Piliackas, J.M., L.M. Barbosa \& E.L.M. Catharino. 2000. Levantamento das epífitas vasculares do manguezal do Rio Picinguaba, Ubatuba, São Paulo. Anais, V Simpósio de Ecossistemas Brasileiros: Conservação. ACIESP 109: 2. 
Pimm, S.L. 2001. The World According to Pimm: a scientist audits the earth. McGraw-Hill, New York, New York, USA.

Pinto, A.C., M.E.S.P. Demattê \& M.C.M.D. Pavani. 1995. Composição florística de epífitas (Magnoliophyta) em fragmento de floresta no município de Jaboticabal, SP, Brazil. Cientifica 22: 283-289.

Silva, S.M. 1998. As formações vegetais da planície litorânea da Ilha do Mel, Paraná: composição florística e principais características estruturais. Ph.D. Thesis. Universidade Estadual de Campinas. Campinas, Brazil.

Sudgen, A.M. \& R.J. Robins. 1979. Aspects of the ecology of vascular epiphytes in Colombian cloud forests, I. distribution of the epiphytic flora. Biotropica 11: 173-188.
Takhtajan, A. 1986. Floristic Regions of the World. University of California, Berkeley, California, USA.

Waechter, J.L. 1986. Epífitos vasculares da mata paludosa do Faxinal, Torres, Rio Grande do Sul, Brazil. Iheringia 34: 39-49.

Waechter, J.L. 1992. O epifitismo vascular na planície costeira do Rio Grande do Sul. Ph.D. Thesis. Universidade Federal de São Carlos, São Carlos, Brazil.

Waechter, J.L. 1998a. Epifitismo vascular em uma floresta de restinga do Brasil Subtropical. Rev. Ciên. Nat. 20: 43-66.

Waechter, J.L. 1998b. Epiphytic Orchids in eastern subtropical America. Proc. World. Conference 15: 332-341. 